\title{
Moisture Diagnosis of Transformer Oil- Immersed Insulation with Intelligent Technique and Frequency-Domain Spectroscopy
}

\author{
Jiefeng Liu, Member, IEEE, Xianhao Fan, Student Member, IEEE, Chaohai Zhang, Member, \\ IEEE, Chun Sing Lai, Senior Member, IEEE, Yiyi Zhang, Member, IEEE, Hanbo Zheng, Loi \\ Lei Lai, Fellow, IEEE, and Enze Zhang
}

\begin{abstract}
Moisture is one of the critical factors to determine the service life of transformers. The moisture inside the transformer oil-immersed insulation could be quantified with feature parameters. This paper proposes and develops a Genetic Algorithm Support Vector Machine (GA-SVM) model to carry out the moisture diagnosis. Present findings reveal that these feature parameters can be obtained by using frequency domain spectroscopy. Therefore, a novel model for predicting frequency domain spectroscopy curves is firstly reported based on a small number of samples, which could be utilized to obtain the feature parameters database to develop GA-SVM. Then, the moisture diagnosis in lab and field conditions is presented to verify its feasibility and accuracy. The novelty of this work is in an exploration of the reported model, as an intelligent based moisture diagnosis tool for power transformers.
\end{abstract}

Index Terms- Power transformer, genetic algorithm support vector machine (GA-SVM), oil-immersed insulation, frequency domain spectroscopy (FDS), moisture diagnosis.

\section{INTRODUCTION}

$\mathrm{T}_{\mathrm{t}}$ he satisfactory operating condition and performance of transformers are essential for a reliable power grid operation. The condition diagnosis and monitoring of oil/paper systems in the transformer are thus of great interest to engineers and researchers. The Dissolved Gas Analysis (DGA) [1] is generally considered to be the most convenient and common approach for reconditioning the energized transformers. Since the diagnostic accuracy of the traditional DGA can no longer meet the requirements of modern power grids, the artificial intelligence diagnostic algorithm based on DGA has become a prevailing method for fault diagnosis of oil/paper system in power transformers [2-3].

The Support Vector Machine (SVM) [4] contains excellent learning and application capacity for solving the linear or

Manuscript received April 06, 2020; revised July 04, 2020, accepted July 26, 2020. Date of publication XXX; date of current version XXX. This work was supported by the Basic Ability Improvement Project for Young and Middle-aged Teachers in Universities of Guangxi 20190046 and 20190067; Brunel Research Initiative and Enterprise Fund (BRIEF); Education Department of Guangdong Province 2016KCXTD022; National Natural Science Foundation of China 51867003 and 61473272; Natural Science Foundation of Guangxi Province 2018JJA160176 and 2018JJB160064. Paper no. TII-20-1713. (Corresponding authors: Chaohai Zhang, Chun Sing Lai and Loi Lei Lai)

J. Liu, X. Fan, C. Zhang, Y. Zhang, H. Zheng and E. Zhang are with the Department of Electrical Engineering, Guangxi University, Guangxi, nonlinear classification. Reviewing the existing researches, SVM can be used to carry out transformer fault diagnosis research based on different databases, such as Partial Discharge (PD) data and DGA data [5-7]. Besides, literature [8] used Genetic Algorithm (GA) for optimizing the DGA ratios and SVM parameters. The fault diagnosis results obtained by the modified (optimized) SVM will keep higher accuracy than three traditional approaches, including back-propagation neural network SVM with DGA data, International Electrotechnical Commission (IEC) criteria, and back-propagation neural network SVM with IEC three-key gas ratios. Thus, the SVM modified by optimization algorithm (such as GA) and Crossvalidation (CV) may be used as an alternative tool for accurate diagnosis on the transformer.

In contrast with the transformer (transient) fault, the insulation performance of the transformer oil/paper system has also attracted great interest since it could determine the service life of the transformer [9-10]. The insulation performance of the oil-paper system is mainly determined by its aging degree [11] and damp state (moisture level) [9]. Moisture is the main byproduct generated by cellulose aging, which is the key factor that aggravates aging activity and shortens the service life of transformer oil-immersed insulation. Specifically, I. The cellulose insulation life will be reduced by half for every $0.5 \%$ increase of moisture [12]; II. The dissolved moisture will be evaporated and floats in the form of bubbles [13], which reduces the breakdown voltage and further leads to the breakdown faults. Reviewing the works from [14-17], the prevailing methods for moisture diagnosis can be divided into online (cellulose-water adsorption isotherms, moisture equilibrium charts, water activity probes) and offline (Karl Fischer titration, dielectric response) approach. Furthermore, the dielectric response is preferable because the uncertainties due to non-equilibrium conditions and oil conditions are reduced [15]. References $[9,16]$ reveal that the Frequency

530004, China (e-mail: liujiefeng9999@163.com; xianhao_fan@163. com; euv@qq.com; yiyizhang@gxu.edu.cn; hanbozheng@163.com; enze_zhang@163.com).

C. S. Lai is with Brunel Institute of Power Systems, Department of Electronic \& Computer Engineering, Brunel University London, London, UB8 3PH, U.K. and also with the Department of Electrical Engineering, School of Automation, Guangdong University of Technology, Guangzhou, 510006, China (e-mail: chunsing.lai@ brunel.ac.uk).

L. L. Lai is with the Department of Electrical Engineering, School of Automation, Guangdong University of Technology, Guangzhou, 510006, China (email: I.I.lai@ieee.org). 
Domain Spectroscopy (FDS) is non-destructive and sensitive enough to the moisture inside the paper/pressboard insulation. Thus, the feature parameters extracted from FDS can be used as an effective indicator for the preliminary diagnosis of its damp states. However, the existing methods mainly rely on fitting functions [9, 16] and grey relational analysis [17], which faced to the limitations of simple classification, no generalization ability, and difficulty in extrapolation. Feature parameters can be used to reflect the inside moisture of transformer oilimmersed insulation. Such parameters could be extracted with FDS or Artificial Intelligence (AI) such as SVM. The trained SVM can be applied to efficiently and accurately carry out the moisture diagnosis. Unfortunately, as for the difficulties of the sample preparation [9], it is a rather difficult task to obtain a large number of samples to form a training set that aims to develop the SVM.

Given this issue, according to the power function theory [18] and dielectric physics theory $[19,20]$, the novel model for predicting FDS curves is firstly reported based on small amount samples. Present findings reveal that the reported model can predict the FDS curves of oil-immersed insulation with diverse moisture, and also obtain the database of feature parameters to develop GA-SVM. The moisture diagnosis in lab and field conditions is presented to verify its feasibility and accuracy. In that respect, a novel method is reported for combining $\mathrm{AI}$ and the FDS technique for power transformer moisture diagnosis.

\section{EXPERIMENTAL Scheme AND FDS TeSt}

\section{A. Preparation of Oil-immersed Cellulose Insulation}

The oil-immersed cellulosic pressboards are obtained by a series of experiments in the controlled laboratory conditions. In this section, pressboard discs (thickness: $1 \mathrm{~mm}$, diameter: 160 $\mathrm{mm}$ ) and transformer oil are utilized. The oil-paper mass ratio is set to 20:1. The transformer oil is the Karamay No.25 naphthenic mineral oil and satisfies the standard of ASTM D3487-2000(II). Where, the oil-immersed insulation samples are prepared by the experiments of vacuum drying (at $105^{\circ} \mathrm{C}$ and $50 \mathrm{~Pa}$ ) and vacuum immersion (at $60^{\circ} \mathrm{C}$ and $50 \mathrm{~Pa}$ ) with the oil paper ratio of $20: 1$. Then, the pressboards with various expected moisture content $(m c \%)$ are obtained by placing it on a precision electronic balance to absorb moisture. Then, the initial moisture is measured by the moister tester (Karl Fischer titration [21] according to IEC 60814), as shown in Fig. 1. Combined with the classification standards (CIGRE TB 349, IEEE 62-1995, and IEC 60422) of $m c \%$ and the requirements of this work (construction of multiple classifiers), the classification table of the damp state is thus defined according to the expected moisture, as is shown in Table I.

TABLE I

Definition of Moisture LeVel of Prepared SAMples.

\begin{tabular}{cccc}
\hline Number & Moisture level & Defined states & State label \\
\hline 1 & $0.5 \% \leq m c \%<1.0 \%$ & Well dry & M1 \\
2 & $1.0 \% \leq m c \%<1.5 \%$ & Dry & M2 \\
3 & $1.5 \% \leq m c \%<2.0 \%$ & Slightly damped & M3 \\
4 & $2.0 \% \leq m c \%<2.5 \%$ & Damped & M4 \\
5 & $2.5 \% \leq m c \%<3.0 \%$ & Moderately damped & M5 \\
6 & $3.0 \% \leq m c \%<3.5 \%$ & Severely damped & M6 \\
7 & $3.5 \% \leq m c \%<4.0 \%$ & Extremely damped & M7 \\
\hline
\end{tabular}

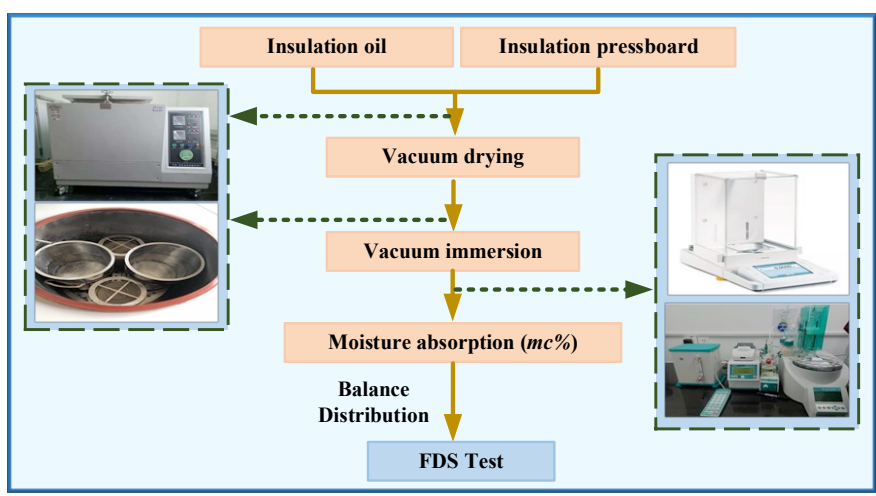

Fig. 1. The experimental scheme for obtaining the oil-immersed cellulose insulation with various moisture in lab condition.

\section{B. Experimental Platform for FDS Test}

The oil-immersed insulation with various moisture levels is considered for performing the FDS test. The test was performed with the device as shown in Fig. 2 which includes the DIRANA/OMICRON and a three-electrode test cell. The test voltage is at $200 \mathrm{VAC}$ and the test temperature preset $45^{\circ} \mathrm{C}$.

The three-electrode test cell is filled with dried and degassed insulating oil. The cellulose paperboard is immersed in the insulating oil under the vacuum. Subsequently, the moisture equilibrium is performed at $45^{\circ} \mathrm{C}$ and lasted for 48 hours before the FDS test. Finally, the measured complex capacitance $C^{*}(\omega)$ of these oil-immersed pressboards (shown in Fig. 2) with different moisture are readily obtained. Then, the real part $\varepsilon^{\prime}(\omega)$ and the imaginary part $\varepsilon^{\prime \prime}(\omega)$ of the complex permittivity can be computed.
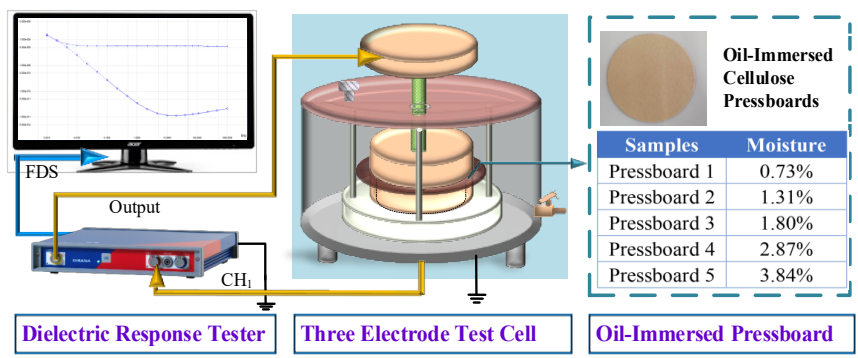

Fig. 2. The schematic of the FDS test.

\section{Establishment of Database for Predicting the FDS CURVES UNDER VARIOUS MOISTURE LEVELS}

The frequency dielectric modulus- $M^{*}(\omega)$ is introduced here for performing the decoupling analysis of FDS of oil-immersed insulation, which can be utilized to establish an available database for computing the corresponding $M^{*}(\omega)$ curves under different moisture levels.

\section{A. Calculation of Frequency Dielectric Modulus}

The electrode polarization and conductance can "obscure" the relaxation information. Hence, the frequency dielectric modulus- $M^{*}(\omega)$ is therefore applied to enable the investigation of the relaxation behavior, as well as highlight the details of the relaxation information in low-frequency regions [19, 22].

According to the existing work [22], the $M^{*}(\omega)$ is defined as the inversion of $\varepsilon^{*}(\omega)$ which is shown in Eq. (1). 


$$
\begin{aligned}
M^{*}(\omega) & =\frac{1}{\varepsilon^{*}(\omega)}=\frac{1}{\varepsilon^{\prime}(\omega)-i \cdot \varepsilon^{\prime \prime}(\omega)} \\
& =\frac{\varepsilon^{\prime}(\omega)+i \cdot \varepsilon^{\prime \prime}(\omega)}{\left[\varepsilon^{\prime}(\omega)-i \cdot \varepsilon^{\prime \prime}(\omega)\right] \cdot\left[\varepsilon^{\prime}(\omega)+i \cdot \varepsilon^{\prime \prime}(\omega)\right]} \\
& =\frac{\varepsilon^{\prime}(\omega)+i \cdot \varepsilon^{\prime \prime}(\omega)}{\varepsilon^{\prime}(\omega)^{2}+\varepsilon^{\prime \prime}(\omega)^{2}}
\end{aligned}
$$

The percentage of the real part $M^{\prime}(\omega)$ and imaginary part $M^{\prime \prime}(\omega)$ of $M^{*}(\omega)$ can be therefore calculated by Eq. (2).

$$
\left\{\begin{array}{l}
M^{\prime}(\omega)=\frac{\varepsilon^{\prime}(\omega)}{\varepsilon^{\prime}(\omega)^{2}+\varepsilon^{\prime \prime}(\omega)^{2}} \times 100 \% \\
M^{\prime \prime}(\omega)=\frac{\varepsilon^{\prime \prime}(\omega)}{\varepsilon^{\prime}(\omega)^{2}+\varepsilon^{\prime \prime}(\omega)^{2}} \times 100 \%
\end{array}\right.
$$

According to Eq. (2), either the $M^{\prime}(\omega)$ or $M^{\prime \prime}(\omega)$ are both calculated by the combination of $\varepsilon^{\prime}(\omega)$ and $\varepsilon^{\prime \prime}(\omega)$.

Such property allows the obtained $M^{*}(\omega)$ can be regarded as a dimensionless value (a kind of ratio value), which is similar to the dispersion loss $(\tan \delta)$. Besides, $M^{*}(\omega)$ can be readily calculated with $\varepsilon^{*}(\omega)$ by using Eq. (2). $M^{*}(\omega)$, i.e. $M^{\prime}(\omega)$ and $M^{\prime \prime}(\omega)$, is presented in Fig. 3 as a log-log model.
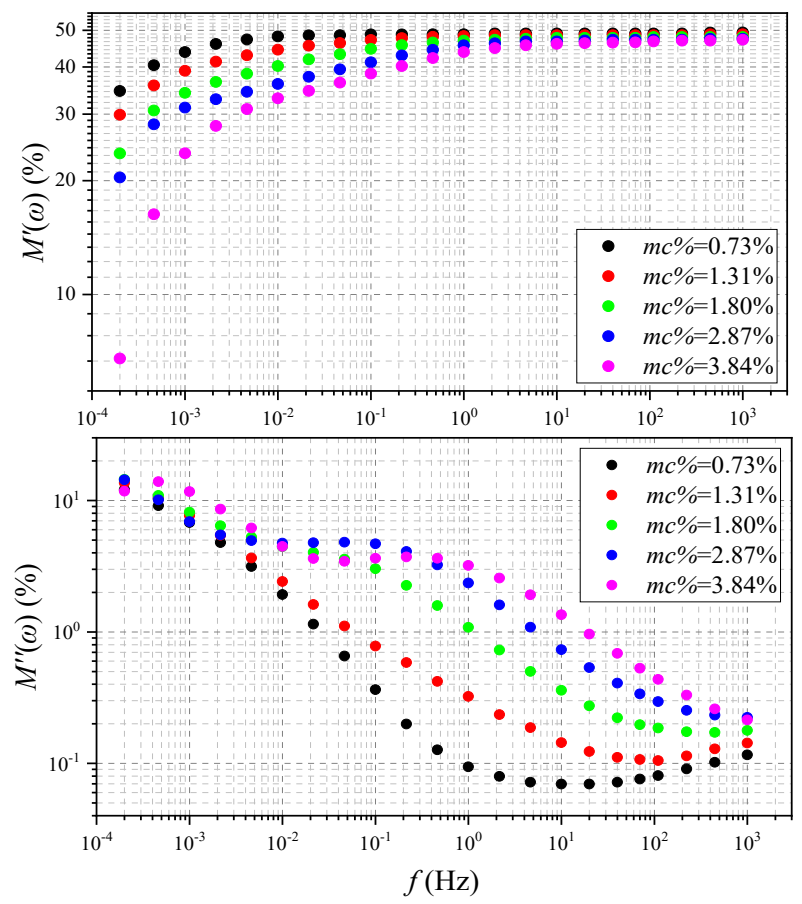

Fig. 3. Real/imaginary part of the dielectric modulus of prepared oilimmersed pressboards.

\section{B. Decoupling Analysis of Frequency Dielectric Modulus}

The widely accepted view suggests that the real part $\varepsilon^{\prime}(\omega)$ and imaginary part $\varepsilon^{\prime \prime}(\omega)$ of the complex permittivity could be utilized to represent the feature of stored energy and dielectric loss, respectively, as shown in Eq. (3) [19].

$$
\left\{\begin{array}{l}
\varepsilon^{\prime}(\omega)=\varepsilon_{\infty}+\frac{\varepsilon_{s}-\varepsilon_{\infty}}{1+(\omega \tau)^{2}} \\
\varepsilon^{\prime \prime}(\omega)=\frac{\left(\varepsilon_{s}-\varepsilon_{\infty}\right) \cdot \omega \tau}{1+(\omega \tau)^{2}}
\end{array}\right.
$$

Where, $\varepsilon_{0}$ is the vacuum permittivity, and $\varepsilon_{0} \approx 8.854 \times 10^{-12}$ $\mathrm{F} / \mathrm{m}$. $\varepsilon_{\infty}$ is the permittivity when angular velocity $\omega \rightarrow \infty$, and $\varepsilon_{s}$ is the static permittivity, the relaxation time constant is $\tau$, denotes the time required by dipoles to rearrange themselves after the exciting electric field is removed. Then, Eq. (4) can be obtained by eliminating $(\omega \tau)$ presented in Eq. (3) [23].

$$
\left[\varepsilon^{\prime}(\omega)-\frac{\left(\varepsilon_{s}+\varepsilon_{\infty}\right)}{2}\right]^{2}+\varepsilon^{\prime \prime}(\omega)^{2}=\frac{\left(\varepsilon_{s}-\varepsilon_{\infty}\right)^{2}}{4}
$$

If the relaxation time distribution parameter $\beta(\beta<1)$ is considered, then the formula of the Cole-Cole model is obtained by the complex transformation $\varepsilon^{*}(\omega)=\varepsilon^{\prime}(\omega)-i \varepsilon^{\prime \prime}(\omega)$ as shown in Eq. (5).

$$
\varepsilon^{*}(\omega)=\varepsilon_{\infty}+\frac{\varepsilon_{r e}}{1+(i \omega \tau)^{\beta}}
$$

Where, $\varepsilon r e^{=} \varepsilon_{s}-\varepsilon_{\infty}$. According to Eq. (1), the formula of $M^{*}(\omega)$ involves the Cole-Cole model. Eq. (1) is transformed to Eq. (6) [22].

$$
\begin{aligned}
& \frac{1}{\varepsilon^{*}(\omega)}=\frac{1}{\varepsilon_{\infty}+\left(\varepsilon_{s}-\varepsilon_{\infty}\right) /\left[1+(i \omega \tau)^{\beta}\right]} \\
& \left.=\frac{1}{\varepsilon_{\infty}} \cdot \frac{1+\frac{\varepsilon_{s} / \varepsilon_{\infty}-1}{1+(i \omega \tau)^{\beta}}-\frac{\varepsilon_{s} / \varepsilon_{\infty}-1}{1+(i \omega \tau)^{\beta}}}{1+\left(\varepsilon_{s} / \varepsilon_{\infty}-1\right) /\left[1+(i \omega \tau)^{\beta}\right]}=\frac{1}{\varepsilon_{\infty}}-\frac{\frac{1}{\varepsilon_{\infty}}-\frac{1}{\varepsilon_{s}}}{1+(i \omega \tau)^{\beta} \cdot\left(\varepsilon_{\infty} / \varepsilon_{s}\right)}\right) \\
& =M_{\infty}+\frac{M_{s}-M_{\infty}}{1+\left(i \omega \tau_{M}\right)^{\beta}}=M^{*}(\omega)
\end{aligned}
$$

Where, $M_{\infty}=1 / \varepsilon_{\infty}, M_{s}=1 / \varepsilon_{s}$, and $\tau_{M}=\tau \cdot\left(\varepsilon_{\infty} / \varepsilon_{s}\right)^{1 / \beta}$. Considering the second relaxation process and DC conductance effect, Eq. (6) can be rewritten as Eq. (7).

$$
M^{*}(\omega)=M_{\infty}+\frac{M_{r e 1}}{1+\left(i \omega \tau_{M 1}\right)^{\beta_{1}}}+\frac{M_{r e 2}}{1+\left(i \omega \tau_{M 2}\right)^{\beta_{2}}}+\frac{\sigma_{d c}}{i \varepsilon_{0} \omega}
$$

Where, $M_{r e i}$ represents the intensity of relaxation polarization and is $M_{s}$ minus $M_{\infty} . \sigma_{d c}$ is the DC conductivity. Complex transformation is applied to obtain the equations for describing the $M^{\prime}(\omega)$ and $M^{\prime \prime}(\omega)$.

$$
\left\{\begin{array}{l}
M^{\prime}(\omega)=M_{\infty}+\frac{M_{r e 1}}{A_{1}} \cos \left(\theta_{1}\right)+\frac{M_{r e 2}}{A_{2}} \cos \left(\theta_{2}\right) \\
M^{\prime \prime}(\omega)=\frac{M_{r e 1}}{A_{1}} \sin \left(\theta_{1}\right)+\frac{M_{r e 2}}{A_{2}} \sin \left(\theta_{2}\right)+\frac{\sigma_{d c}}{\varepsilon_{0} \omega}
\end{array}\right.
$$

Where, $A_{i}$ and $\theta_{i}(i=1,2)$ can be expressed by Eq. (9).

$$
\left\{\begin{array}{l}
A_{i}=\sqrt{\left[1+\left(\omega_{i} \tau_{M i}\right)^{\beta_{i}} \cos \left(\frac{\beta_{i} \pi}{2}\right)\right]^{2}+\left[\left(\omega_{i} \tau_{M i}\right)^{\beta_{i}} \sin \left(\frac{\beta_{i} \pi}{2}\right)\right]^{2}} \\
\theta_{i}=\arctan \left\{\left[\left(\omega_{i} \tau_{M i}\right)^{\beta_{i}} \sin \left(\frac{\beta_{i} \pi}{2}\right)\right] /\left[1+\left(\omega_{i} \tau_{M i}\right)^{\beta_{i}} \cos \left(\frac{\beta_{i} \pi}{2}\right)\right]\right\}
\end{array}\right.
$$

From Eq. (8), the value of $M^{*}(\omega)\left[M^{\prime}(\omega)\right.$ or $\left.M^{\prime \prime}(\omega)\right]$ is restricted by an obvious multivariate nonlinear function, which depends on the parameter set $\left\{M_{\infty}, M_{r e 1}, \beta_{1}, \tau_{M 1}, M_{r e 2}, \beta_{2}, \tau_{M 2}\right.$, $\left.\sigma_{d c}\right\}$. Thus, if a certain function $(\Psi)$ is found to be able to simulate the $M^{*}(\omega)$ curves, the decoupling analysis can be therefore achieved by using the defined parameters. Such a functional relationship can be represented by Eq. (10).

$$
M^{*}(\omega)=\Psi\left[M_{\infty}, M_{r e 1}, \beta_{1}, \tau_{M 1}, M_{r e 2}, \beta_{2}, \tau_{M 2}, \sigma_{d c}\right]
$$

The frequency dielectric response behavior could be summarized by the superposition of several sub-relaxation processes with diverse relaxation time constants. 
Therefore, on the continuous curves, the value of $M^{*}(\omega)$ in each frequency point can be regarded as the superposition of different functional items according to the power function theory [18].

In this work, the power series $(\Omega)$ is utilized to achieve this goal, since it is capable of exploring the functional relationship of $M^{\prime}(\omega)$ or $M^{\prime \prime}(\omega)$ versus defined parameters. Besides, the order of the power series equals to the number of variables contained in the functional equation $(\Psi)$. The function $\Psi$ can be revised as shown by Eq. (11).

$$
\begin{aligned}
& M^{*}(\omega)=\Psi\left[M_{\infty}, M_{r e 1}, \beta_{1}, \tau_{M 1}, M_{r e 2}, \beta_{2}, \tau_{M 2}, \sigma_{d c}\right] \\
& \Rightarrow \Omega\left[\Theta_{0}, \Theta_{1}, \Theta_{2}, \Theta_{3}, \Theta_{4}, \Theta_{5}, \Theta_{6}, \Theta_{7}\right] \\
&=\Theta_{0}+\Theta_{1} \cdot\left(\omega-\omega_{0}\right)+\Theta_{2} \cdot\left(\omega-\omega_{0}\right)^{2}+\cdots+\Theta_{7} \cdot\left(\omega-\omega_{0}\right)^{7}
\end{aligned}
$$

Where, $j$ is a series of function terms, $\Theta_{0}$ is the intercept of power functions, the $\Theta_{j}(j=1-7)$ is defined as the amplitude of each sub-function. According to Eq. (11), the value of $M^{*}(\omega)$ is defined by eight parameters $\left\{M_{\infty}, M_{\mathrm{re} 1}, \beta_{1}, \tau_{M 1}, M_{\mathrm{re} 2}, \beta_{2}, \tau_{M 2}\right.$, $\left.\sigma_{\mathrm{dc}}\right\}$, therefore, preset the value of 0 to 7 as the region of $j$ to define the initial condition, then, parameters $\Theta_{j}$ can be used to carry out the fitting analysis.

Theoretically, a larger $j$ value provides a better goodness-offit measure. However, results indicated that it is not a good choice to have $j$ greater than 7 , due to the increasing uncertainty of the fitting analysis. Especially, if the initial sampling frequency is low enough and close to 0 , then $\omega_{0}$ equals to 0 as shown in Eq. (12).

$$
\begin{aligned}
& M^{*}(2 \pi f)=\Theta_{0}+\Theta_{1} \cdot(2 \pi f)+\Theta_{2} \cdot(2 \pi f)^{2}+\cdots+\Theta_{7} \cdot(2 \pi f)^{7} \\
& \Rightarrow M^{*}(f)=\sum_{j=0}^{7} \Theta_{j} \cdot(f)^{j}
\end{aligned}
$$

$M^{*}(\omega)$ conveys the frequency-dependence and is also affected to the moisture level. Consequently, Eq. (12) can be revised as Eq. (13).

$$
\begin{gathered}
M^{*}(f, m c \%)=\Theta_{0}(m c \%)+\Theta_{1}(m c \%) \cdot f+\Theta_{2}(m c \%) \cdot f^{2} \\
+\cdots+\Theta_{7}(m c \%) \cdot f^{7}=\sum_{j=0}^{7} \Theta_{j}(m c \%) \cdot f^{j}
\end{gathered}
$$

Providing that the FDS data is drawn in the log-log model, as shown in Fig. 3, Eq. (13) can be modified as Eq. (14).

$$
\begin{aligned}
& \lg \left[M^{*}(f, m c \%)\right]=\sum_{j=0}^{7} \Theta_{j}(m c \%) \cdot(\lg f)^{j} \\
& \Rightarrow M^{*}(f, m c \%)=10^{\wedge}\left[\sum_{j=0}^{7} \Theta_{j}(m c \%) \cdot(\lg f)^{j}\right]
\end{aligned}
$$

\section{The Database for Predicting the Frequency Dielectric Modulus Curves under Diverse Moisture}

Fig. 3 shows the moisture information contained in both the real/imaginary part of the dielectric modulus. $M^{\prime}(\omega)$ characterizes the polarization information inside the dielectric, and its microcosmic response mechanism is relatively simple. $M^{\prime \prime}(\omega)$ characterizes the dielectric loss information, including the polarization loss and the conductivity loss. Therefore, its microcosmic response mechanism is more complicated than $M^{\prime}(\omega)$. The increasing moisture causes the $M^{\prime}(\omega)$ curve in the low-frequency regions to rise while the high-frequency regions remain essentially unchanged. In this case, the moisture level slightly changes the original shape of the $M^{\prime}(\omega)$ curve, which makes it simplifies the decoupling analysis, as well as more efficient to explore the functional relationship between $\Theta_{j}$ and the moisture.

Eq. (14) and Fig. 3 will enable the fitting analysis of the measured discrete points of $M^{\prime}(\omega)$. As discussed, the fitting accuracy is satisfactory when $j$ reaches 6 , while, the goodnessof-fit measure reaches the best when $j=7$. Therefore, $j=7$ is selected to fitting $M^{\prime}(\omega)$ curves and extract the relevant parameters. The fitting curves corresponding to these discrete points can be later obtained, as well as the series of parameters contained in the equation. Fig. 4 presents the comparison of the measured $M^{\prime}(\omega)$ and its fitting curves when $j$ equals to 3,7 , and 8 , respectively. Table II depicts the parameters $\left(\Theta_{0}-\Theta_{7}\right)$ contained in Eq. (14).

Besides, the average value of goodness-of-fit measure $\left(\mathrm{R}^{2}\right)$ between the measured value and fitting curves is 0.9589 , 0.9997 , and 0.9997 , respectively, when $j$ equals to 3, 7, and 8 . As the $\mathrm{R}^{2}$ value is high, the confidence band will coincide with the fitting curves and cannot be distinguished, the $95 \%$ confidence level is thus omitted and only $\mathrm{R}^{2}$ is presented.

From Table II, the contained parameters $\left(\Theta_{0}-\Theta_{7}\right)$ alter regularly with the changing internal moisture, and the values of these parameters are considered to determine the original shape of fitting curves.

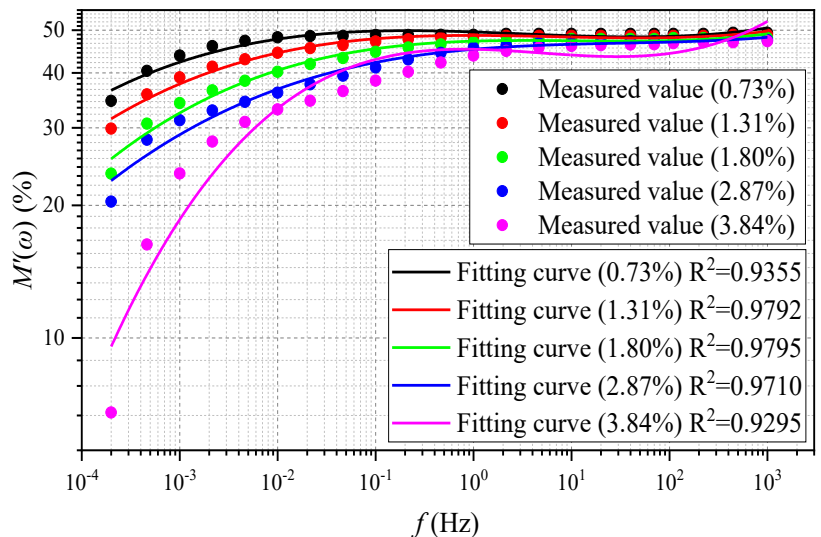

(a)

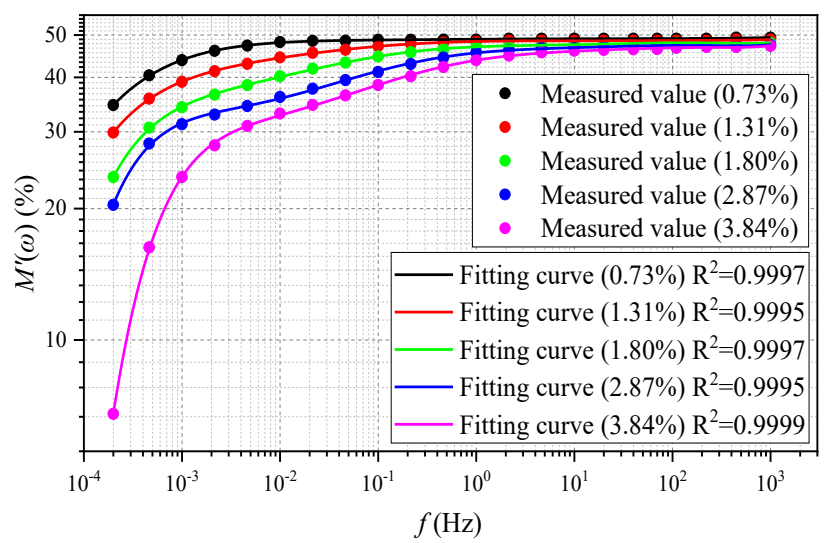

(b) 


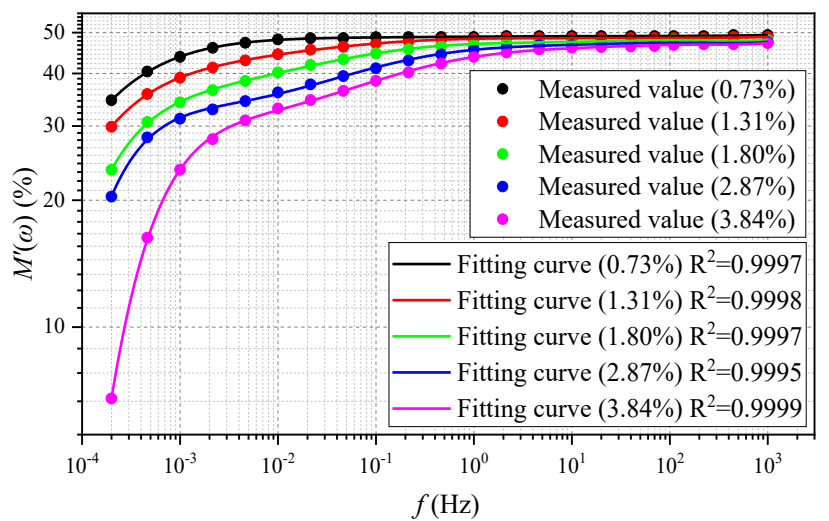

(c)

Fig. 4. The comparison of the measured $M^{\prime}(\omega)$ and its fitting curves. (a) $j=3$, (b) $j=7$, (c) $j=8$.

TABLE II

The Value of Parameters Contained in Fitting Curves.

\begin{tabular}{c|ccccc}
\hline \multirow{2}{*}{ Parameters } & \multicolumn{5}{|c}{ Moisture content (\%) } \\
& $0.73 \%$ & $1.31 \%$ & $1.80 \%$ & $2.87 \%$ & $3.84 \%$ \\
\hline$\Theta_{0}$ & 1.6895 & 1.6847 & 1.6730 & 1.6593 & 1.6425 \\
$\Theta_{1}$ & 0.0015 & 0.0048 & 0.0108 & 0.0240 & 0.0380 \\
$\Theta_{2}$ & $-5.07 \mathrm{E}-4$ & -0.0059 & -0.0112 & -0.0191 & -0.0236 \\
$\Theta_{3}$ & $-1.82 \mathrm{E}-4$ & 0.0017 & 0.0036 & 0.0039 & 0.0016 \\
$\Theta_{4}$ & $5.18 \mathrm{E}-5$ & $5.32 \mathrm{E}-4$ & 0.0012 & 0.0026 & 0.0041 \\
$\Theta_{5}$ & $8.75 \mathrm{E}-5$ & $-1.55 \mathrm{E}-4$ & $-4.92 \mathrm{E}-4$ & $-7.70 \mathrm{E}-4$ & $-6.37 \mathrm{E}-4$ \\
$\Theta_{6}$ & $-2.98 \mathrm{E}-5$ & $-4.73 \mathrm{E}-5$ & $-8.37 \mathrm{E}-5$ & $-1.49 \mathrm{E}-4$ & $-3.21 \mathrm{E}-4$ \\
$\Theta_{7}$ & $2.13 \mathrm{E}-6$ & $1.16 \mathrm{E}-5$ & $2.87 \mathrm{E}-5$ & $4.56 \mathrm{E}-5$ & $6.78 \mathrm{E}-5$ \\
\hline
\end{tabular}

To verify this viewpoint and investigate the potential variation law of $\Theta_{j}(j=0-7)$ versus moisture, the fitting analysis is conducted. The obtained equation for quantitatively describing the relationship between $\Theta_{j}$ and moisture is written as Eq. (15). Besides, the comparison of $\Theta_{j}$ and the fitting curves is provided in Fig. 5. The filled color area near the fitting curve is the confidence band of the parameter at $95 \%$ confidence level.

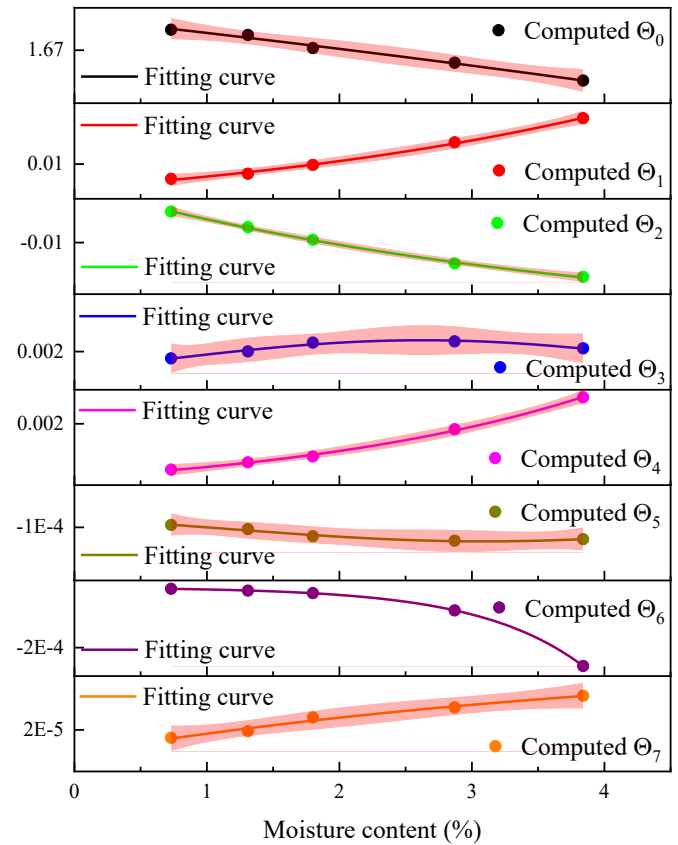

Fig. 5. The comparison of the calculated $\Theta_{j}$ and the fitting curves.

$$
\begin{cases}\Theta_{0}=1.956-0.255 \cdot \operatorname{EXP}(m c \% / 18.429) & \mathrm{R}^{2}=0.991 \\ \Theta_{1}=-0.024+0.020 \cdot \operatorname{EXP}(m c \% / 3.463) & \mathrm{R}^{2}=0.998 \\ \Theta_{2}=-0.038-0.045 \cdot \operatorname{EXP}(-m c \% / 3.851) & \mathrm{R}^{2}=0.999 \\ \Theta_{3}=-0.011+0.016 \cdot \operatorname{EXP}\left[-0.5 \times\left(\frac{m c \%-2.631}{2.041}\right)^{2}\right] & \mathrm{R}^{2}=0.988 \\ \Theta_{4}=-0.001+8.9 \mathrm{E}-4 \cdot \operatorname{EXP}(m c \% / 2.389) & \mathrm{R}^{2}=0.999 \\ \Theta_{5}=0.001-0.002 \cdot \operatorname{EXP}\left[-0.5 \times\left(\frac{m c \%-3.095}{1.973}\right)^{2}\right] & \mathrm{R}^{2}=0.982 \\ \Theta_{6}=-2.0 \mathrm{E}-5-1.8 \mathrm{E}-6 \cdot \operatorname{EXP}(m c \% / 0.790) & \mathrm{R}^{2}=0.999 \\ \Theta_{7}=1.8 \mathrm{E}-4-2.1 \mathrm{E}-4 \cdot \operatorname{EXP}(-m c \% / 4.551) & \mathrm{R}^{2}=0.983\end{cases}
$$

With Fig. 5 and Eq. (15), the defined $\Theta_{j}$ can be calculated when substituting the given moisture. Thus, the predicted $M^{\prime}(\omega)$ curves under diverse moisture $m c_{i} \%$ can be represented by a matrix formed with parameters $\Theta_{i j}$, as shown in Eq. (16).

$$
\left[\begin{array}{c}
M_{1}^{\prime}\left(\omega, m c_{1} \%\right) \\
M_{2}^{\prime}\left(\omega, m c_{2} \%\right) \\
\mathrm{M} \\
M_{i}^{\prime}\left(\omega, m c_{i} \%\right)
\end{array}\right] \Leftrightarrow\left[\begin{array}{ccc}
\Theta_{11}\left(m c_{1} \%\right), & \Theta_{12}\left(m c_{1} \%\right), \mathrm{L}, \Theta_{17}\left(m c_{1} \%\right)^{-} & \\
\Theta_{21}\left(m c_{2} \%\right) & \Theta_{22}\left(m c_{2} \%\right), \mathrm{L}, \Theta_{27}\left(m c_{2} \%\right) & (16 \\
\mathrm{M} & \mathrm{M} & \mathrm{M} \\
\Theta_{i 1}\left(m c_{i} \%\right), & \Theta_{i 2}\left(m c_{i} \%\right), \mathrm{L}, \Theta_{i 7}\left(m c_{i} \%\right)
\end{array}\right.
$$

Considering that there are seven parameters in the above matrix of parameters, each set of $M^{\prime}(\omega)$ curves with different moisture can be predicted by this database.

The value of $M^{\prime}(\omega)$ at each sampling point can be predicted point-by-point until a continuous curve is formed by using Eq. (15) and Eq. (16).

Fig. 6 shows the relationship between the moisture level, frequency, and $M^{\prime}(\omega)$ in the $x$-axis, $y$-axis, and $z$-axis, respectively. The figure shows a smooth surface can be constructed with more $M^{\prime}(\omega)$ curves. Such a database can be utilized for obtaining a large number of defined parameters $\Theta_{i j}$ and $M^{\prime}(\omega)$ curves under various moisture levels.

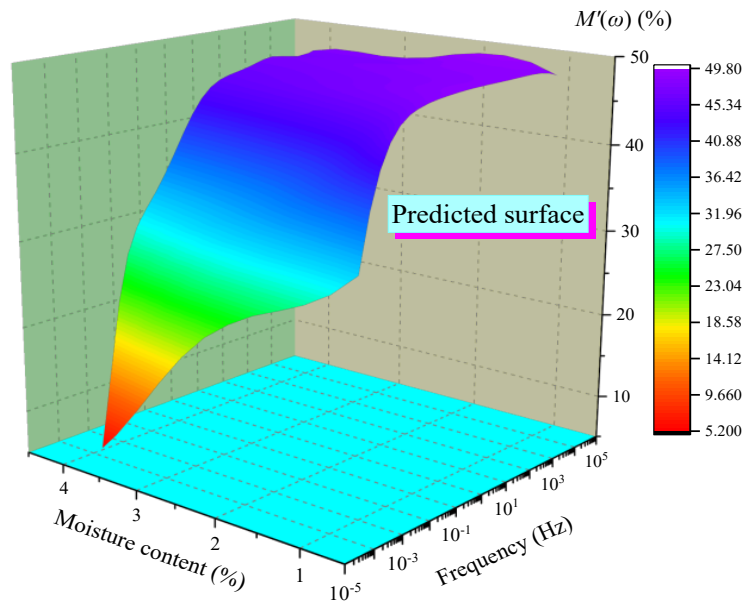

Fig. 6. The fitting database for extracting the feature parameters.

\section{Application of InTELligence Algorithm For MOISTURE DIAGNOSIS}

\section{A. Application of GA-SVM}

GA-SVM is introduced to perform parameter optimization by using GA. Its classification principle can be expressed by Eq. (17) [4]. 


$$
\begin{aligned}
& \min _{\min } \Phi\left(\omega, \xi_{i}\right)=\frac{1}{2}\|\omega\|^{2}+c \sum_{i=1}^{n} \xi_{i} \\
& \text { s.t. }\left\{\begin{array}{l}
y_{i}\left(\omega^{T} x_{i}+b\right) \geq 1-\xi_{i} \\
\xi_{i} \geq 0 \quad(i=1,2 \cdots n)
\end{array}\right.
\end{aligned}
$$

Where, $\xi_{i}$ is the slack variable and $c$ is the penalty factor. When $\Phi\left(\omega, \xi_{i}\right)$ reaches the minimum value, the margin between positive and negative examples reaches the maximum, and the determined decision surface is exactly the Optimal Separating Hyperplane (OSH). The optimization condition of Eq. (17) can be confirmed by Eq. (18).

$$
\begin{aligned}
& L\left(\omega, \xi_{i}, b, \alpha_{i}, \beta_{i}\right) \\
& =\frac{1}{2}\|\omega\|^{2}+c \sum_{i=1}^{n} \xi_{i}-\sum_{i=1}^{n} \alpha_{i}\left[y_{i}\left(\omega^{T} x_{i}+b\right)-1+\xi_{i}\right]-\sum_{i=1}^{n} \beta_{i} \xi_{i}
\end{aligned}
$$

Parameters $\alpha_{i}$ and $\beta_{i}$ are introduced by Lagrange number multiplication.

Eq. (17) can be revised as Eq. (20) when Eq. (18) satisfies the optimization conditions as shown in Eq. (19).

$$
\begin{gathered}
\partial L / \partial \omega=0 \Rightarrow \omega=\sum_{i=1}^{n} \alpha_{i} y_{i} x_{i} \\
\partial L / \partial \xi_{i}=0 \Rightarrow c-\alpha_{i}-\beta_{i}=0 \\
\partial L / \partial b=0 \Rightarrow \sum_{i=1}^{n} \alpha_{i} y_{i}=0 \\
\max \Psi(\alpha)=\sum_{i=1}^{n} \alpha_{i}-\frac{1}{2} \sum_{i, j=1}^{n} \alpha_{i} \alpha_{j} y_{i} y_{j} K\left(x_{i}, x_{j}\right) \\
\text { s.t. } 0 \leq \alpha_{i} \leq c, \sum_{i=1}^{n} \alpha_{i} y_{i}=0, K\left(x_{i}, x_{j}\right)=\phi\left(x_{i}\right) \cdot \phi\left(x_{j}\right)
\end{gathered}
$$

Where, $\phi(x)$ belongs to a mapping operation and $K\left(x_{i}, x_{j}\right)$ is called the kernel function. $K\left(x_{i}, x_{j}\right)$ allows the inner product that should be performed in the high-dimensional space to be completed in the input space.

The radial basis kernel function shown in Eq. (21) is selected since it is capable of optimizing the generalization performance of GA-SVM.

$$
K\left(x_{i}, x_{j}\right)=\operatorname{EXP}\left(-\gamma\left\|x_{i}-x_{j}\right\|^{2}\right), \gamma>0
$$

Where, $\gamma$ is the key parameter of the radial basis kernel function. Solving the parameter $\alpha_{i}$ from Eq. (20) and include it into Eq. (22), the decision function for classifying is obtained.

$$
f(x)=\operatorname{sign}\left[\sum_{i=1}^{n} \alpha_{i} y_{i} K\left(x_{i}, x\right)+b\right]
$$

By relying on the following steps, the performance of condition classification could be improved by GA [6-7].

i. Binary coding the core parameters $(c$ and $\gamma)$ and then constructing the initial population;

ii. Calculating the fitness of each individual in the population by Eq. (23), then taking the classification accuracy of cross-validation of GA-SVM as the fitness value. Where, $n_{1}$ $(n 0)$ is the number of samples with correct (incorrect) classifications;

$$
f=n_{1} /\left(n_{1}+n_{0}\right)
$$

iii. Once meeting the termination condition, decompile and output the best parameters $c$ and $\gamma$. If not, proceed to the next step;

iv. Optimizing the parents with genetic operators (selection, crossover, mutation). Generate offspring and return to step ii.

\section{B. Multi-classifier for Moisture Diagnosis using GA-SVM}

From Table II, the parameters $\Theta_{j}$ are highly correlated with the moisture. Thus, the combination of $\Theta_{i j}$ could form a database and further utilized for training the multi-classifier of moisture diagnosis with GA-SVM. Fig. 7 summarizes the corresponding steps for establishing the multi-classifier.

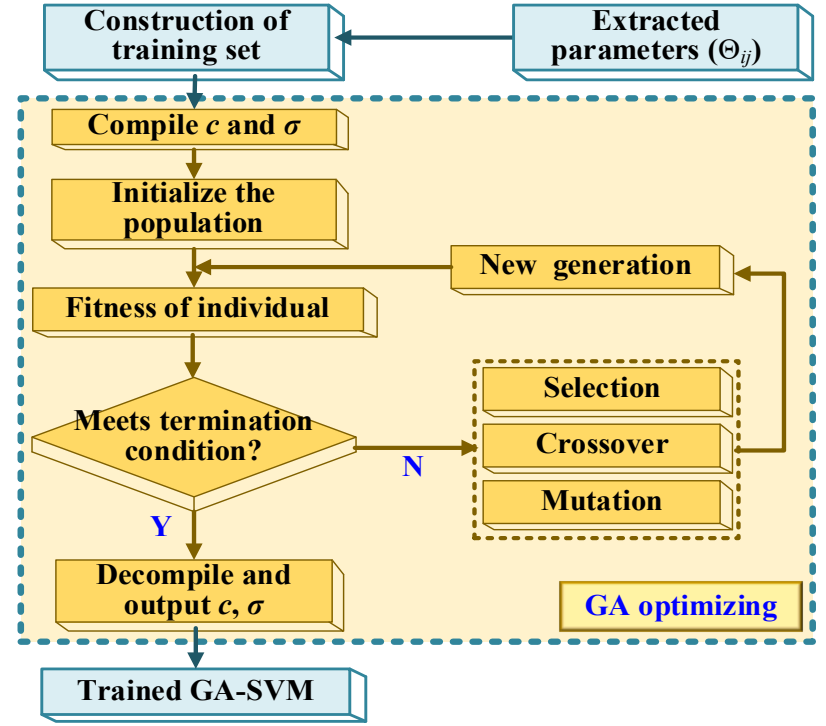

Fig. 7. Multi-classifier based on the GA-SVM.

\section{i. Discovery of parameters used for classification}

As to Eq. (16), the contained parameters $\Theta_{i j}$ convey the strong correlation with the inside moisture. Thus, samples with the given moisture level $m c_{i} \%$ could determine a parameter set $\Omega_{i}$, the inside parameters $\Theta_{i j}$ can be used to classify the diverse damp states.

ii. Construction of GA-SVM training set

If the classification result of GA-SVM is required to be accurate enough, the required samples must be provided as a training set in advance. However, in practical experiments, it is a rather difficult task to prepare a large number of oil-immersed samples with different moisture due to factors such as accuracy, duration, and consumables of samples preparation. Therefore, a database (shown in Fig. 6) is proposed to overcome the above issues.

Specifically, the preset type of damp states is 7, as is shown in Table I. The range of moisture level starts at $0.5 \%$ and ends at $4 \%$ with $0.01 \%$ increment. Therefore, 351 parameter sets $\left(\Omega_{i}\right)$ can be calculated by Eq. (15), and further applied for classifying the 7 kinds of damp states $\left(M_{1}-M_{7}\right)$. Consequently, the 2808 parameters $\left(\Theta_{i j}\right)$ from 351 parameter sets $\left(\Omega_{i}\right)$ can be utilized to build the training set of 7 kinds of damp states $\left(M_{1}-M_{7}\right)$ for training GA-SVM. 
iii. Parameter optimization and multi-classifier construction

To improve the accuracy of classification results, Genetic Algorithm is used to optimize the model parameters $c$ and $\gamma$. From Fig. 7, the Genetic Algorithm continuously adjusts the constituent of the parameter population according to its fitness, and then removes the individuals with lower fitness. Thus, the number of high-quality individuals in the population will increase as the calculation cycle increases.

Fig. 8 shows that the average fitness fluctuates slightly while the best fitness remains unchanged until iterates to 100 generations. Moreover, the cross-validation accuracy of GASVM in the training set reaches $99.15 \%$. The obtained parameters $(c=1.1745$ and $g=98.7512)$ is a near global optimal solution, which can be used to optimize the accuracy of multiclassification. Therefore, the database (developed by Eq. (16)) provides the parameters to construct the training set, and the GA-SVM multi-classifier is built for moisture diagnosis.

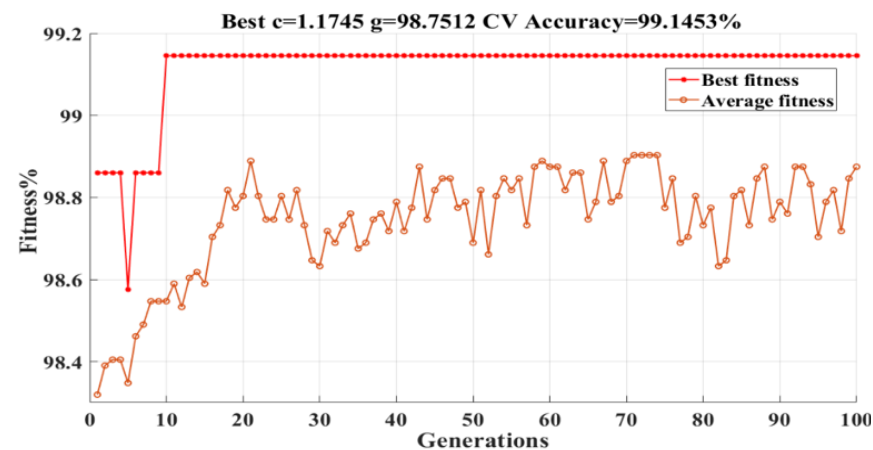

Fig. 8. Fitness curves obtained by GA-SVM.

\section{The Comparison of Classification Accuracy}

Two algorithms are utilized to discuss the comparisons with GA-SVM, including a. SVM without optimization [4]; b. SVM optimized by Particle Swarm Optimization (PSO-SVM) [3]. The training conditions and training sets are consistent with GA-SVM for a fair comparison. Fig. 9 shows the classification results of SVM and PSO-SVM. The average classification accuracy of the SVM and PSO-SVM are $97.43 \%$ and $99.15 \%$ respectively. Thus, the AI algorithm could improve the classification accuracy of the SVM. The optimization of the reported model is not only limited to the use of the GA but also other similar AI algorithms.

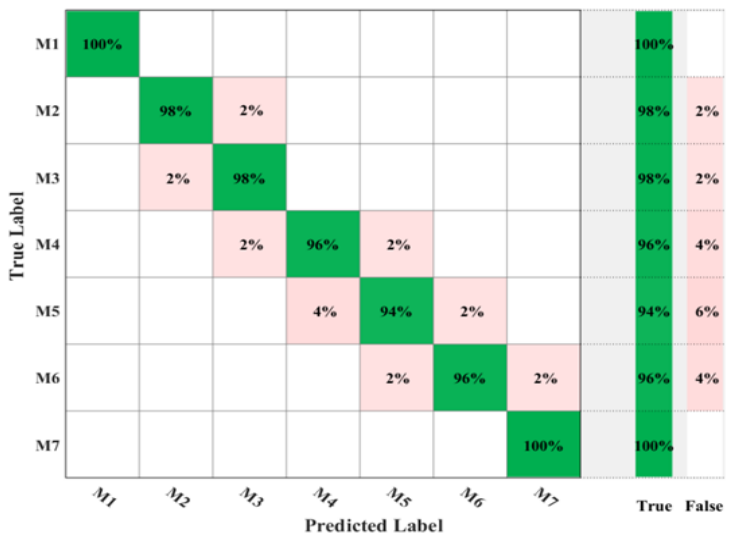

(a)

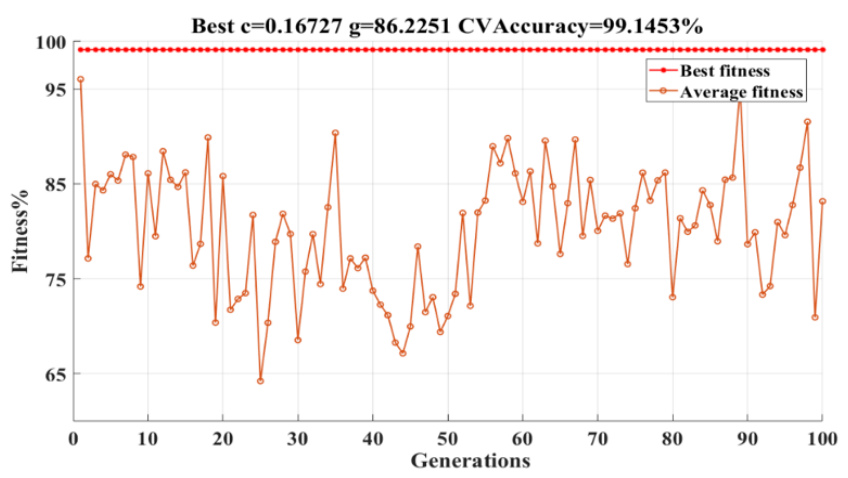

(b)

Fig. 9. The classification accuracy. (a) SVM, (b) PSO-SVM.

\section{Application of the Proposed Model for Moisture DiAGNOSIS IN LAB AND FIELD CONDITIONS}

\section{A. Application in Lab Conditions}

In order to perform the moisture diagnosis, the FDS data of test samples should be obtained in advance. Subsequently, the established multi-classifier based on SVM and GA-SVM (shown in Section IV) can be later utilized to perform the moisture diagnosis.

Meanwhile, a series of new samples with various moisture levels are prepared and used to verify the feasibility and accuracy of the reported model. The detailed scheme for feasibility verification is as follows:

i. First, the complex capacitance of test samples was measured. Then, the complex relative permittivity is calculated;

ii. Second, the corresponding $M^{\prime}(\omega)$ is calculated by Eq. (2), and the feature parameters $\left(\Theta_{i j}\right)$ is extracted by using Eq. (14), and $i \in(1,5)$. The extracted values are shown in Eq. (24);

$\left[\begin{array}{l}\Theta_{i 0}\left(m c_{i} \%\right) \\ \Theta_{i 1}\left(m c_{i} \%\right) \\ \Theta_{i 2}\left(m c_{i} \%\right) \\ \Theta_{i 3}\left(m c_{i} \%\right) \\ \Theta_{i 4}\left(m c_{i} \%\right) \\ \Theta_{i 5}\left(m c_{i} \%\right) \\ \Theta_{i 6}\left(m c_{i} \%\right) \\ \Theta_{i 7}\left(m c_{i} \%\right)\end{array}\right]=\left[\begin{array}{ccccc}1.6891 & 1.6778 & 1.6658 & 1.6546 & 1.6445 \\ 0.0014 & 0.0076 & 0.0156 & 0.0244 & 0.0337 \\ -9.24 \mathrm{E}-4 & -0.0075 & -0.0131 & -0.0017 & -0.0201 \\ 1.83 \mathrm{E}-4 & 0.0035 & 0.0054 & 0.0052 & 0.0036 \\ -1.88 \mathrm{E}-6 & 4.65 \mathrm{E}-4 & 0.0011 & 0.0019 & 0.0029 \\ 3.69 \mathrm{E}-5 & -5.13 \mathrm{E}-4 & -9.65 \mathrm{E}-4 & -0.0011 & -0.0010 \\ -2.47 \mathrm{E}-5 & -3.29 \mathrm{E}-5 & -5.53 \mathrm{E}-5 & -1.06 \mathrm{E}-4 & -2.07 \mathrm{E}-4 \\ 5.17 \mathrm{E}-6 & 3.17 \mathrm{E}-5 & 5.47 \mathrm{E}-5 & 7.23 \mathrm{E}-5 & 8.55 \mathrm{E}-5\end{array}\right]$

iii. Finally, the diagnosis results are obtained by substituting the parameters $\left(\Theta_{i j}\right)$ into SVM and GA-SVM.

Fig. 10 presents the comparison of measured $M^{\prime}(\omega)$ and fitting curves. According to Table I, the Measured States (MS, by Karl Fischer titration [21]) and Diagnosis States (DS, by using SVM and GA-SVM) are tabulated in Table III.

TABLE III

DiAgNOSIS RESULTS OF TESTED SAMPLES.

\begin{tabular}{c|cccccc}
\hline Samples & 1 & 2 & 3 & 4 & 5 & WR \\
\hline MS (by KFT) & M1 & M3 & M4 & M6 & M7 & $/$ \\
\hline DS (by SVM) & M1 & M3 & M4 & M7 & M7 & 1 \\
DS (GA-SVM) & M1 & M3 & M4 & M6 & M7 & 0 \\
\hline
\end{tabular}




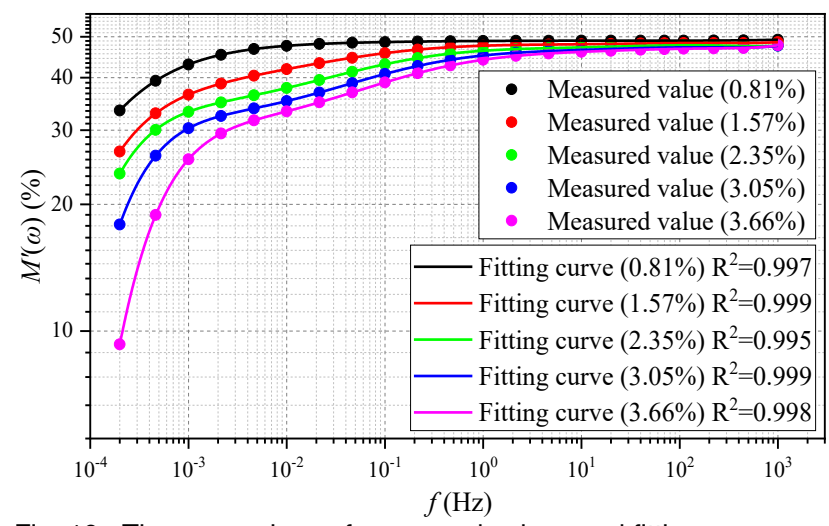

Fig. 10. The comparison of measured values and fitting curves.

The wrong result (WR) obtained by SVM and GA-SVM is 1 and 0 , respectively. Thus, the accuracy of the classifier optimized by the AI algorithm is higher.

\section{B. Application in Field Conditions}

The 3-winding transformers under maintenance are selected, its detailed parameters are shown in Table IV. The main insulation system between the high and medium voltage winding is measured with the help of DIRANA during the FDS test, the scheme of field application is presented in Fig. 11 and described as follows:

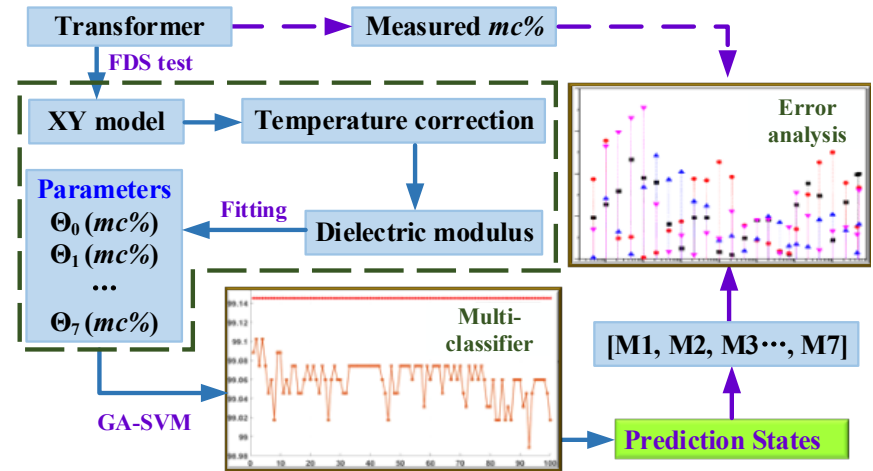

Fig. 11. The scheme for moisture diagnosis in field testing.

TABLE IV

The Extracted Parameters of Tested Samples.

\begin{tabular}{c|ccc}
\hline Parameters & Trans.1 & Trans.2 & Trans.3 \\
\hline Voltage classes & $110 \mathrm{kV}$ & $110 \mathrm{kV}$ & $220 \mathrm{kV}$ \\
Loading history & 1 year & 8 years & 8 years \\
Oil temperature & $303.15 \mathrm{~K}$ & $302.15 \mathrm{~K}$ & $300.15 \mathrm{~K}$ \\
Oil & $12 \mathrm{fS} / \mathrm{m}$ & $63 \mathrm{fS} / \mathrm{m}$ & $280 \mathrm{fS} / \mathrm{m}$ \\
conductivity & & $\mathrm{X}=0.29$, & $\mathrm{X}=0.19$, \\
XY value & $\mathrm{X}=0.27, \mathrm{Y}=0.20$ & $\mathrm{Y}=0.24$ & $\mathrm{Y}=0.14$ \\
Shift factor & $\alpha_{\mathrm{T}}=6.49$ & $\alpha_{\mathrm{T}}=7.40$ & $\alpha_{\mathrm{T}}=9.65$ \\
Tested moisture & $0.7 \%$ & $1.2 \%$ & $1.3 \%$ \\
\hline
\end{tabular}

i. The complex capacitance $C_{t o t} *(\omega)$ of the selected field transformer is measured. Fig. 12 presents the connection of the FDS test in field transformers. The unit of analysis is the main insulation system between high voltage winding and medium voltage winding;

ii. $\varepsilon_{t o t}^{*}(\omega)$ can be computed by employing the formula of $\varepsilon_{t o t} *(\omega)=C_{t o t} *(\omega) / C_{0} . C_{0}$ is a constant of geometric capacitance;

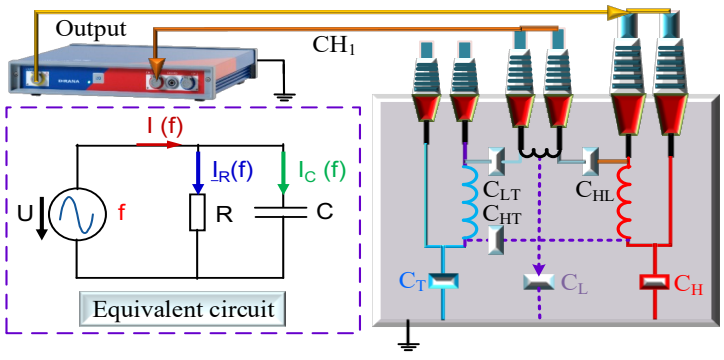

Fig. 12. The connection diagram of the FDS test in field transformers.

iii. To eliminate the influence of the insulation geometry on the measured FDS data, the XY model [24] is utilized to extract the dielectric response data, corresponding to the paper insulation $\varepsilon_{P B}^{*}(\omega)$ from the total FDS data of the main insulation system $\varepsilon_{t o t} *(\omega)$. The XY model is shown in Eq. (25). The $\mathrm{X}$ and $\mathrm{Y}$ values can be found in Table IV, and $\sigma(T)$ is the conductivity of insulating oil at temperature $T$;

$$
\left\{\begin{array}{l}
\varepsilon_{\text {tot }}^{*}(\omega)=\frac{1-\mathrm{Y}}{(1-\mathrm{X}) / \varepsilon_{\text {oil }}^{*}(\omega)+\mathrm{X} / \varepsilon_{P B}^{*}(\omega)}+\mathrm{Y} \times \varepsilon_{P B}^{*}(\omega) \\
\varepsilon_{\text {oil }}^{*}(\omega)=2.2-j \frac{\sigma(T)}{\varepsilon_{0} \omega}
\end{array}\right.
$$

iv. The transformer's main insulation system is difficult to be in a settled temperature field and the temperature can greatly affect its FDS data [10]. If the test temperatures in the field conditions are different from the lab temperatures, then the temperature effect on the extracted FDS data requires correction before performing the moisture diagnosis.

From the previous sections, the reported model is examined at $45^{\circ} \mathrm{C}$ and is different from the oil-temperature of the field test. Eq. (26) is thus applied for eliminating the temperature impact on $\varepsilon P B^{*}(\omega)$ according to the shift factor $\alpha_{T}[10] . E_{a}$ is the activation energy, $E_{a} \approx 100 \mathrm{~kJ} / \mathrm{mol} . R$ is a gas constant and $R=8.314 \mathrm{~J} /(\mathrm{mol} \cdot \mathrm{K}) . T_{\text {ref }}$ is the reference temperature (i.e. $318.15 \mathrm{~K})$ and $T$ is the oil temperature;

$$
\alpha_{T}=\operatorname{EXP}\left[\frac{E_{a}}{R}\left(\frac{1}{T}-\frac{1}{T_{r e f}}\right)\right]
$$

v. $M^{\prime}(\omega)$ of paper insulation under reference temperature can be computed by using the $\varepsilon_{P B} *(\omega)$ (obtained in Step iii) corrected by Eq. (26). The reference temperature (at $45^{\circ} \mathrm{C}$ ) is the approximate average temperature between the oil temperature during the transformer normal operation and the ambient temperature during the off-line measurement. The reference temperature enhances the accuracy of the generalized FDS data and helps shorten the time required for moisture equilibrium. It is possible to choose a different reference temperature to build the proposed model;

vi. Eq. (14) is utilized to extract the relevant parameters $\Theta_{i j}$, as shown in Eq. (27). The comparison of the measured $M^{\prime}(\omega)$ and the fitting curve is plotted in Fig. 13;

$$
\begin{array}{llllllll}
\Theta_{i 0} & \Theta_{i 1} & \Theta_{i 2} & \Theta_{i 3} & \Theta_{i 4} & \Theta_{i 5} & \Theta_{i 6} & \Theta_{i 7}
\end{array}
$$

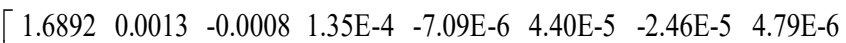
$\begin{array}{llllllll}1.6834 & 0.0044 & -0.0045 & 2.00 \mathrm{E}-3 & 2.19 \mathrm{E}-4 & -2.47 \mathrm{E}-4 & -2.79 \mathrm{E}-5 & 1.94 \mathrm{E}-5\end{array}$ $\begin{array}{llllllll}1.6804 & 0.0061 & -0.0062 & 2.85 \mathrm{E}-3 & 3.48 \mathrm{E}-4 & -3.92 \mathrm{E}-4 & -3.03 \mathrm{E}-4 & 2.62 \mathrm{E}-5\end{array}$ 


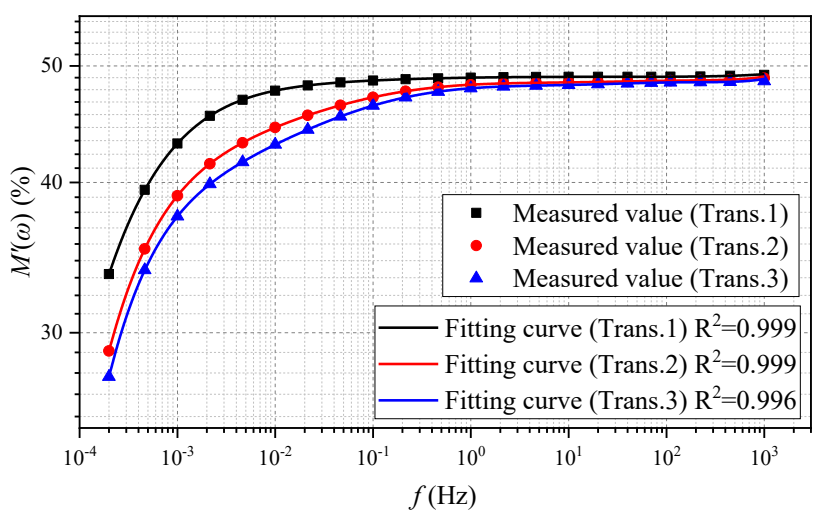

Fig. 13. The comparison of measured values and fitting curves.

vii. The extracted parameters $\left(\Theta_{i j}\right)$ shown in Eq. (27) are the diagnostic basis for moisture classification when employing the proposed moisture classifier. DS obtained by the trained multiclassifier (SVM and GA-SVM) is tabulated in Table V, as well as its measured states (MS, by DIRANA).

TABLE V

The Diagnosis Results of Tested Transformers.

\begin{tabular}{cccc}
\hline Transformers & Trans. 1 & Trans. 2 & Trans. 3 \\
\hline MS (by DIRANA) & M1 & M2 & M2 \\
\hline DS (by SVM) & M1 & M3 & M7 \\
DS (by GA-SVM) & M1 & M2 & M2 \\
\hline
\end{tabular}

According to Table I, the difference of moisture content between two adjacent states $(\mathrm{M} i)$ is $0.5 \%$. In Tables III and V, the classification error of Sample 4 (in Table III) and Trans. 2 (in Table V) reveals that the SVM may not accurately classify the samples with similar moisture as the optimized SVM (GASVM). Also, the classification accuracy of the optimized SVM (GA-SVM) is higher than the SVM.

In summary, the moisture diagnosis results shown in Tables III and V could be utilized to verify the accuracy and feasibility of the proposed method. The experimental results obtained in the lab condition initially show the potential of this method for diagnosis in extreme moisture conditions. The main insulation system and test environment during the field test are more complex than lab conditions. Hence, the accuracy of the obtained results is thus likely to be lower than the lab conditions. However, the contribution of moisture to the FDS data will be amplified with increasing moisture [25]. Meanwhile, moisture will gradually become the dominant factor of dielectric response, which not only increase the proportion of moisture information in FDS, but also promotes the moisture analysis activities. Such assumption deserves further attention.

\section{CONCLUSION}

Therefore, this work proposes to perform the moisture diagnosis for power transformers with GA-SVM and FDS technique. The model is trained with feature parameters (i.e., moisture) computed from FDS. The key challenge is on the preparation of a large number of oil-immersed samples and obtaining the required training set that aims to develop GASVM. Therefore, a novel model for predicting FDS curves is reported. The analysis has led to the following conclusions:
1. The dielectric modulus derived from the complex permittivity can be utilized to perform the decoupling analysis of FDS of transformer, as well as to obtain the feature parameters $\left(\Theta_{i j}\right)$ that aim to represent the moisture content inside the transformer;

2. A novel model is proposed to predict $M^{\prime}(\omega)$ curves under diverse moisture. The model is based on regression analysis and power series theory, and can be trained with small amount of samples. The model can be also utilized to obtain the feature parameters database to develop GA-SVM;

3. The cross-validation accuracy of GA-SVM trained by the obtained feature parameters database reaches $99.15 \%$, which is an effective moisture diagnosis result. In particular, the proposed model outperforms SVM without optimization and similar to other AI models;

4. The presented moisture diagnosis in lab and field conditions verified the feasibility and accuracy of the proposed method. In that respect, the novelty of this work is in an exploration of the reported method by combining AI (GA-SVM) and FDS as a potential and accessible industrial tool for moisture diagnosis of transformer insulation.

At present, the application of this model to site trial is under consideration by power utilities. It is hoped to have some results for reporting in due course. Also, further research could focus on the generalization and the application of the reported method under various test conditions, especially at the extreme damp conditions.

\section{REFERENCES}

[1] R. B. Fabio et al., "Application of an artificial neural network in the use of physicochemical properties as a low cost proxy of power transformers DGA data," IEEE Trans. Dielectr. Electr. Insul., vol. 19, no. 1, pp. 239246, 2012.

[2] Z. Sahri, R. Yusof, and J. Watada, "Finnim: Iterative Imputation of Missing Values in Dissolved Gas Analysis Dataset," IEEE Trans. Ind. Inform., vol. 10, no. 4, pp. 2093-2102, 2014.

[3] K. Wang et al., "A hybrid algorithm based on s transform and affinity propagation clustering for separation of two simultaneously artificial partial discharge sources," IEEE Trans. Dielectr. Electr. Insul., vol. 22 no. 2, pp. 1042-1060, 2015.

[4] A. Jindal et al., "Decision tree and SVM-based data analytics for theft detection in smart grid," IEEE Trans. Ind. Inform., vol. 12, no. 3, pp. 1005-1016, 2016.

[5] Q. Su et al., "A fuzzy dissolved gas analysis method for the diagnosis of multiple incipient faults in a transformer," IEEE Trans. Power Syst., vol. 15, no. 2, pp. 593-598, May 2000.

[6] K. Wang et al., "A new image-oriented feature extraction method for partial discharges," IEEE Trans. Dielectr. Electr. Insul., vol. 22, no. 2, pp. 1015-1024, 2015.

[7] Y. Y. Zhang et al., "A fault diagnosis model of power transformers based on dissolved gas analysis features selection and improved Krill Herd algorithm optimized support vector machine," IEEE Access, vol. 7, no. 99, pp. 102803-102811, 2019.

[8] J. Li et al., "Optimal dissolved gas ratios selected by genetic algorithm for power transformer fault diagnosis based on support vector machine," IEEE Trans. Dielectr. Electr. Insul., vol. 23, no. 2, pp. 1198-1206, 2016.

[9] J. F. Liu et al., "Quantitative evaluation for moisture content of cellulose insulation material in paper/oil system based on frequency dielectric modulus technique," Cellulose, vol. 27, pp. 2343-2356, 2020.

[10] J. F. Liu et al., "Temperature correction to frequency dielectric modulus and activation energy prediction of immersed cellulose insulation," IEEE Trans. Dielectr. Electr. Insul., vol. 27, no. 3, pp. 956-963, 2020.

[11] S. Thakur et al., "Thermal aging of cellulosic pressboard material and its surface discharge and chemical characterization," Cellulose, vol. 24, pp. 5197-5210, 2017.

[12] N. Lelekakis, D. Martin, and J. Wijaya, “Ageing rate of paper insulation 
used in power transformers Part 2: Oil/paper system with medium and high oxygen concentration," IEEE Trans. Dielectr. Electr. Insul., vol. 19, no. 6, pp. 2009-2018, 2012.

[13] J. Hill et al., "Analysing the power transformer temperature limitation for avoidance of bubble formation," High Voltage, vol. 4, no. 3, pp. 210-216, 2019.

[14] T. V. Oommen, "Moisture equilibrium charts for transformer insulation drying practice," IEEE Trans. Power App. Syst., vol. 103, pp. 3063-3067, 1984.

[15] W. S. Zaengl, "Applications of dielectric spectroscopy in time and frequency domain for HV power equipment," IEEE Electr. Insul. Mag., vol. 19, no. 6, pp. 9-22, 2003.

[16] L. J. Yang et al., "Assessment of oil-paper insulation aging using frequency domain spectroscopy and moisture equilibrium curves," IEEE Access, vol. 7, pp. 45670-45678, 2019.

[17] J. F. Liu et al., "Condition prediction for oil-immersed cellulose insulation in field transformer using fitting fingerprint database," IEEE Trans. Dielectr. Electr. Insul., vol. 27, no. 1, pp. 279-287, 2020.

[18] J. Allouche et al., "Hadamard grade of power series," Journal of Number Theory, vol. 131, no. 11, pp. 2013-2022, 2013.

[19] A. K. Jonscher, "Dielectric relaxation in solids," Journal of Physics D: Applied Physics, vol. 32, no. 14, pp. 57-70, 1999.

[20] A. K. Jonscher, "Low-frequency dispersion in carrier dominated dielectric," Philosophical Mag. Part B, vol. 38, no. 6, pp. 587-601, 1968.

[21] J. Mitchell, "Karl Fischer reagent titration," Anal. Chem., vol. 23, no. 8, pp. 1069-1075, 1951.

[22] F. Q. Tian, and Y. Ohki, "Electric modulus powerful tool for analyzing dielectric behavior," IEEE Trans. Dielectr. Electr. Insul., vol. 2, no. 3, pp. 929-931, 2014.

[23] K. S. Cole, R. H. Cole, "Dispersion and absorption in dielectrics-I: alternating current characteristics," J. Chem. Phys, vol. 9, pp. 341-351, 1941.

[24] T. K. Saha and P. Purkait, "Investigations of temperature effects on the dielectric response measurements of transformer oil-paper insulation system," IEEE Trans. Power Del., vol. 23, no. 1, pp. 252-260, 2008.

[25] R. B. Jadav, C. Ekanayake, and T. K. Saha, "Understanding the impact of moisture and ageing of transformer insulation on frequency domain spectroscopy," IEEE Trans. Dielectr. Electr. Insul., vol. 21, no. 1, pp. 369-379, 2014.

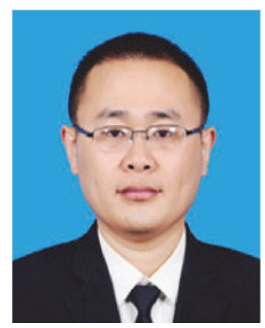

Jiefeng Liu (M'15) received the M.S. and Ph.D. in electrical engineering from Chongqing University, Chongqing, China in 2011 and 2015, respectively. In 2014, He joined Guangxi University where he is an assistant professor. His major research interests include the field of condition assessment and fault diagnosis for oil-paper insulation.

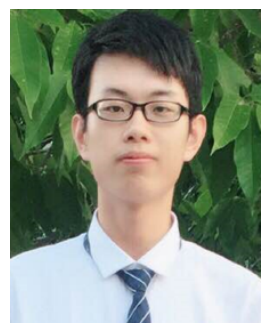

Xianhao Fan (S'18) received the B.E. in electrical engineering in 2018 from Guangxi University. He is currently purchasing a Ph.D. at the Guangxi University, Nanning, China. His current research interests include condition assessment for oil-paper insulation.

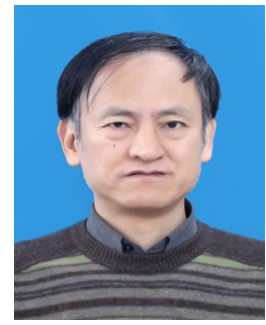

Chaohai Zhang (M'05) received the B.A., M.S and Ph.D. from Harbin Institute of Technology (HIT), Navy Aeronautical Engineering Academy (NAEA) and Hong Kong Polytechnic University (HKPU), respectively. After some years experiences of research working as a JSPS Research Fellow in Kumamoto University, Japan, as a research engineer in Canada, and the chief research scientist in at State Grid EPRI, China, respectively. Currently, he is an adjunct professor of GXU. His research interests are mainly in high voltage engineering, electrical discharges, pulsed power \& plasma application, renewable energy and the condition monitoring, diagnosis, and management of electric power equipment.

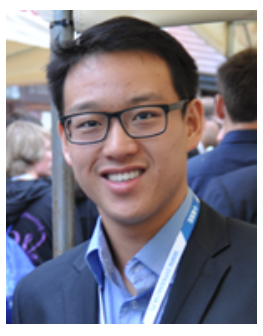

Chun Sing Lai (S'11, M'19, SM'20) received the B.Eng. (First Class Honours) in electrical and electronic engineering from Brunel University London, U.K. and D.Phil. in engineering science from the University of Oxford, U.K. in 2013 and 2019 , respectively. Dr Lai is currently a Lecturer at Department of Electronic and Computer Engineering, Brunel University London, U.K. and also a Visiting Academic with the Department of Electrical Engineering, Guangdong University of Technology, China. From 2018 to 2020, he was an Engineering and Physical Sciences Research Council Research Fellow with the School of Civil Engineering, University of Leeds.

$\mathrm{He}$ is Secretary of the IEEE Smart Cities Publications Committee and Acting EiC of IEEE Smart Cities Newsletters. He is the Publications CoChair for 2020 IEEE International Smart Cities Conference. Dr Lai is the Working Group Chair for IEEE P2814 Standard. His current research interests are in power system optimization and data analytics.

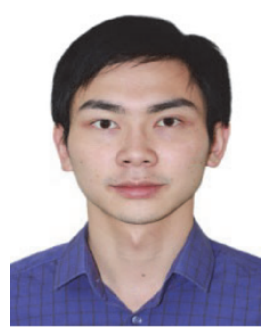

Yiyi Zhang (M'14) received the B.E. and Ph.D. in electrical engineering in 2008 and 2014, respectively from Guangxi University, Nanning, China and Chongqing University, Chongqing, China. In 2014, He joined Guangxi University where he is an associate professor. His current research interests include intelligent diagnosis for transformers.

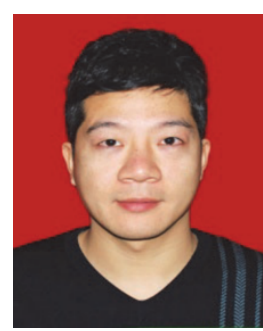

Hanbo Zheng received the M.S. and Ph.D. in electrical engineering from Chongqing University, Chongqing, China in 2009 and 2012, respectively. Dr. Zheng's major research interests include insulation state assessment and fault diagnosis technology for power transformers. 


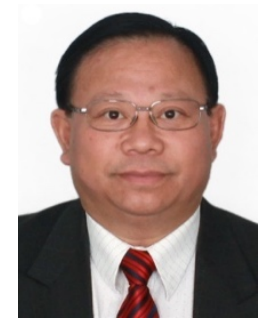

Loi Lei Lai (M'87, SM'92, F'07) received B.Sc. (First Class Honours) in 1980, Ph.D. in 1984 and D.Sc. in 2005 from University of Aston, UK and City, University of London, UK, respectively, all in electrical and electronic engineering. $\mathrm{He}$ is University Distinguished Professor at the Guangdong University of Technology, China. He was Pao Yue Kong Chair Professor, Zhejiang University, China; Director of Research and Development Centre, State Grid Energy Research Institute, China; Vice President for IEEE SMC Society; Professor \& Chair in Electrical Engineering at City, University of London; and a Fellow Committee Evaluator for IEEE IES.

He was awarded IEEE PES UKRI Power Chapter Outstanding Engineer Award in 2000, IEEE PES Energy Development and Power
Generation Committee Prize Paper in 2006 \& 2009, IEEE SMCS Most Active Technical Committee Award in 2016. He is a Fellow of IET. His current research areas are in smart cities and smart grid.

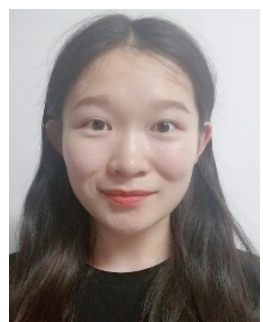

Enze Zhang was born in Inner Mongolia, China in 1996. She is currently pursuing the Ph.D. with the Electrical Engineering Department, Guangxi University of China. Her major research interests include insulation state assessment and fault diagnosis technology for large power transformers. 